Check for updates

Cite this: RSC Adv., 2017, 7, 44568

Received 8th August 2017

Accepted 9th September 2017

DOI: $10.1039 / c 7 r a 08774 h$

rsc.li/rsc-advances

\section{Direct catalytic effect of nitrogen functional groups exposed on graphenic materials when acting cooperatively with Ru nanoparticles $\uparrow$}

\author{
Carolina Ramirez-Barria, ${ }^{\text {ab }}$ Cristina López-Olmos, ${ }^{\text {b }}$ Antonio Guerrero-Ruiz (D) *ac \\ and Inmaculada Rodríguez-Ramos iD bc
}

\begin{abstract}
A number of inorganic carbonaceous materials (activated carbon, high surface area graphite and graphenic materials) have been used as supports of Ru nanoparticles in order to determine their catalytic properties in the base-free aqueous-phase oxidation of 5-hydroxymethylfurfural (HMF) to 2,5-furandicarboxylic acid (FDCA). In particular, we have studied in detail reduced graphene oxide ( $\mathrm{KGO}$ ) and nitrogen doped reduced graphene oxide ( $\mathrm{NGO}$ ), which are the support materials that produce more selective ruthenium catalysts. Also the effects of different metal precursors used in the preparation of the Ru nanocrystallites have been evaluated. Both support materials and Ru catalysts were characterized by elemental analysis, nitrogen physisorption (BET), thermogravimetric analysis (TGA), transmission electron microscopy (TEM), and X-ray photoelectron spectroscopy (XPS). The point of zero charge (PZC) for the graphenic materials was also determined. Interestingly the different supports significantly modify the catalytic performances, the graphenic materials being those that under our experimental reaction conditions produce the highest selectivity to FDCA. On these supports ( $\mathrm{rGO}$ and $\mathrm{NrGO}$ ) the highest HMF conversion was achieved by using triruthenium dodecacarbonyl as the ruthenium precursor. For the improved catalyst, Ru supported on NrGO, the yield of FDCA becomes close to $80 \%$. This catalyst has been reused several times with neither loss of activity nor modification in selectivity values. Characterization data indicate these catalytic results can be correlated to the basic properties of the NrGO support as well as to the surface properties of Ru nanoparticles. These findings indicated that the metal precursor and the surface functional groups exposed on the support can modulate the catalytic properties, in particular amending the selectivity towards FDCA production.
\end{abstract}

\section{Introduction}

In the last few years there has been a growing interest in the conversion of renewable biomass resources into chemicals and fuels. ${ }^{1}$ In this context, HMF is a promising biomass-derived platform molecule generally produced through chemical dehydration of hexoses, such as glucose and fructose. ${ }^{2}$ The oxidation of HMF can generate several kinds of products such as 2,5diformylfuran (DFF), 5-hydroxymethyl-2-furancarboxylic acid (HFCA), 5-formyl-2-furancarboxylic acid (FFCA) and 2,5-

${ }^{a}$ Dpto. Química Inorgánica y Técnica, Facultad de Ciencias UNED, Senda del Rey 9, 28040 Madrid, Spain. E-mail: aguerrero@ccia.uned.es

${ }^{b}$ Instituto de Catálisis y Petroleoquímica, CSIC, Cantoblanco, Marie Curie 2, 28049 Madrid, Spain

${ }^{c}$ Unidad Asociada UNED-ICP/CSIC Grupo de Diseño y Aplicación de Catalizadores Heterogéneos, Spain

$\dagger$ Electronic supplementary information (ESI) available: Fig. S1 contains TEM micrographs of the different graphenic supports, Fig. S2 contains XPS spectra of the $\mathrm{N}$ 1s region for NrGO, Fig. S3 contains TGA for graphenic supports and Fig. S4 XPS spectra of the $\mathrm{Ru} 3 \mathrm{p}$ region of $\mathrm{Ru}(\mathrm{Cl}) / \mathrm{rGO}$ and $\mathrm{Ru}(\mathrm{Cl}) / \mathrm{NrGO}$ catalysts. See DOI: 10.1039/c7ra08774h furandicarboxylic acid (FDCA) (Scheme 1). FDCA has been identified by the U.S. Department of Energy as one of the 12 top value added chemicals from biomass. ${ }^{3}$ FDCA can be used as an alternative monomer to potentially replace terephthalic acid which is involved in the production of polyethylene terephthalate (PET). ${ }^{4}$

Several stoichiometric oxidants, such as $\mathrm{HNO}_{3}, \mathrm{~N}_{2} \mathrm{O}_{4}$ and $\mathrm{KMnO}_{4},{ }^{5}$ as well as homogenous catalytic systems ${ }^{6}$ have been used for the HMF oxidation into FDCA. However, harsh reaction conditions, corrosive properties of the media and production of large amounts of waste have a negative economic and environmental impact.

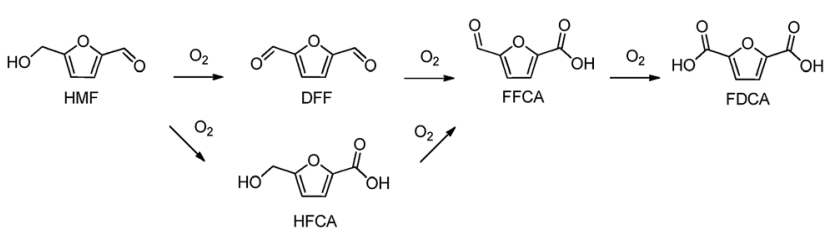

Scheme 1 Reaction pathway from HMF to FDCA. 
The oxidation of HMF to synthesize FDCA employing air or oxygen has been described along with different supported catalysts. ${ }^{7}$ Among the studied heterogeneous catalysts are noteworthy those based on noble metals such as $\mathrm{Pt},{ }^{\mathbf{8} 9} \mathrm{Au},{ }^{\mathbf{1 0 , 1 1}}$ and Ru. ${ }^{12}$ However, it should be underlined that in most of the reported reaction procedures a base additive, essentially $\mathrm{NaOH}$, $\mathrm{KOH}$ or $\mathrm{Na}_{2} \mathrm{CO}_{3}$, is required, ${ }^{\mathbf{1 3 - 1 5}}$ so only very few works applying base-free catalysts have been reported.

Casanova et al. ${ }^{16}$ claimed a useful strategy to avoided base added requirement, using methanol as solvent instead of water. Using an $\mathrm{Au} / \mathrm{CeO}_{2}$ catalyst under oxygen pressure, 2,5-dimethylfuroate is the main product. Despite its outstanding activity and selectivity, the oxidation of the solvent was unavoidable due to it follows a similar oxidation pathway than the substrate.

Highly basic solid supports have also been proposed as replacements of the base during HMF oxidation in water. Gupta and coworkers ${ }^{17}$ reported $\mathrm{Au}$ nanoparticles supported on hydrotalcite (HT) to catalyze the aqueous-phase oxidation of HMF to FDCA showing good performances. However, later studies ${ }^{18}$ suggested that alkali contaminants from the synthesis of the HT supports or co-existing partially soluble brucite favored the results obtained. Along the same lines, high yields of FDCA were also obtained by Gorbanev et al. ${ }^{19}$ using $\mathrm{Ru}(\mathrm{OH})_{X}$ supported on a HT and MgO. Nevertheless, extensive deactivation due to leaching of magnesium from both supports is taking placed. In recent publications $\mathrm{Au}-\mathrm{Pd}$ alloys ${ }^{20}$ and $\mathrm{Pt}^{21}$ supported on functionalized carbon nanotubes (CNT) were presented as base-free alternatives in the aerobic oxidation of HMF to FDCA under oxygen pressure. It is reported that the oxygen-containing functional groups, in particular carbonyl/quinone and/or phenol groups, on CNT surfaces play crucial roles in FDCA formation. These functional groups could enhance the adsorption of HMF as well as the reaction intermediates from water and might facilitate hydrogen transfer. Nonetheless, long reactions times of 12 and $14 \mathrm{~h}$ respectively were required besides the use of expensive metals as Au, Pt and Pd. Also, the facet effect and size-dependent effect of single-crystalline Pd nanocrystals on the aerobic oxidation of HMF has been systematically investigated by experimental and theoretical approaches. $^{22}$ It was found that the size-dependent effect of these Pd nanocrystals derived from the different surface Pd atom percentages. So, Pd atoms at (111) facets exhibited notably enhanced catalytic activity for the aerobic oxidation of HMF than Pd atoms at (100) facets. By controlling the amount of surface Pd atoms to be identical, Pd nanocrystals with the same shape but different particle sizes exhibited very similar catalytic performances for HMF oxidation. Ruthenium catalysts supported on carbon materials were studied by Yi et al. ${ }^{23}$ however very high metal : HMF ratios are required to achieve significant catalytic yield to FDCA.

Due to the lack of more economically attractive and stable heterogeneous catalyst systems, we propose the development of new nanomaterials, consisting of nitrogen doped graphene and small Ru crystallites, which must be efficient and stable when acting as heterogeneous catalysts for the aerobic oxidation of HMF into FDCA in the water media. Moreover we aim to work under base-free conditions taking advantage of the surface functional groups incorporated in the support material. Also we are using for this study $\mathrm{Ru}$ based catalysts, as this metal is cheaper than $\mathrm{Au}, \mathrm{Pd}$ and $\mathrm{Pt}^{.24}$

Special consideration will be paid to the effect induced by the surface functional groups exposed at the surfaces of graphenic materials, such as, nitrogen groups which have been reported previously by our group as basic ingredient of catalysts. ${ }^{25}$ More precisely, it has been demonstrated that the incorporation of $\mathrm{N}$ into these graphenic materials improves their chemical and electrical properties. It is due to the fact that $\mathrm{N}$ presence improves electronic density of the carbon material incorporating by one more electron into the carbon surface becoming more basic. ${ }^{26}$ This possibility of acting on the carbon surface properties and the high specific surface area of graphenic materials, make them highly promising materials. So, recently de Jongh et al. ${ }^{11}$ reported that Au nanoparticles are affected by the presence of surface oxygen groups exposed on high surface area graphite when used in the HMF oxidation, but the reaction experiments were carried out in the presence of $\mathrm{NaHCO}_{3}$ solved in the media. Also in a recent review article ${ }^{27}$ has been remarked the interest in controlling and applying the functionalization of graphene materials. However, to the best of our knowledge, there is no report on the using of nitrogen doped graphene materials as support for the base free oxidation of HMF. Herein, we comparatively investigate the preparation and characterization of $\mathrm{Ru}$ based catalysts supported on carbonaceous materials as activated carbon, high surface area graphite, graphenic materials. These catalytic materials have been applied in the base-free oxidation of HMF in water to yield FDCA, aiming to achieve higher reaction yields at moderate reaction conditions (temperature and time in reaction).

\section{Experimental}

\section{Preparation of supports}

Graphenic materials were obtained via thermal treatment of graphite oxide (GO). GO was synthesized from natural graphite powder (325 mesh) supplied by Alfa Aesar (purity 99.8\%) following a modification of the Brodie's method. ${ }^{28}$ This procedure is as follows: $10 \mathrm{~g}$ of graphite $(\mathrm{G})$ were added to $200 \mathrm{~mL}$ of fuming $\mathrm{HNO}_{3}$ kept at $0{ }^{\circ} \mathrm{C}$ in the reaction flask. $80 \mathrm{~g}$ of $\mathrm{KClO}_{3}$ were slowly added during 2 hours. Thereafter, the mixture was stirred for $21 \mathrm{~h}$ maintaining the temperature. The resulting GO was filtered and washed thoroughly with deionized water until neutral pH. The sample was dried overnight at $100{ }^{\circ} \mathrm{C}$. Exfoliation of the synthesized GO was carried out in a vertical quartz reactor under inert and reactive atmospheres. Two exfoliation conditions have been applied. One where GO was heated under nitrogen ( $87 \mathrm{~mL} \mathrm{~min}^{-1}$ ) until $700{ }^{\circ} \mathrm{C}$ (yielding $\mathrm{rGO}$ ) while for the second consists in passing a mixture of $\mathrm{NH}_{3}, \mathrm{H}_{2}$ and $\mathrm{N}_{2}$ with flow rates of 10,3 and $87 \mathrm{~mL} \min ^{-1}$ while the temperature increased up to $700{ }^{\circ} \mathrm{C}$ with a heating rate of $10^{\circ} \mathrm{C} \mathrm{min}{ }^{-1}$ (giving NrGO). Apart from the lab prepared graphenic materials a commercial activated carbon (AC, provided by Oleicola el Tejar, Córdoba Spain, $S_{\mathrm{BET}}=1190 \mathrm{~m}^{2} \mathrm{~g}^{-1}, 313 \mathrm{~m}^{2} \mathrm{~g}^{-1}$ external surface area) and a high surface area graphite (HSAG 400, from 
TIMCAL, $S_{\text {BET }}=396 \mathrm{~m}^{2} \mathrm{~g}^{-1}$ ) were also employed as support of $\mathrm{Ru}$ metallic nanoparticles.

\section{Preparation of catalysts}

All supported $\mathrm{Ru}$ catalyst were prepared in order to obtain samples with ruthenium loading of $4 \mathrm{wt} \%$. Three different precursors were used in the catalyst preparation. $\mathrm{Ru}_{3}(\mathrm{CO})_{12}$ (catalyst series denoted with "CO") was incorporated in the supports by wetness impregnation, once dissolved the exact amount in acetone. The solvent was removed under reduced pressure on a rotary evaporator at about $50{ }^{\circ} \mathrm{C}$ during at least 30 minutes. Before characterization and catalytic tests these materials were treated under hydrogen flow $\left(60 \mathrm{~mL} \mathrm{~min}^{-1}\right)$ at $350{ }^{\circ} \mathrm{C}$ for $2 \mathrm{~h}$, in order to decompose the precursor and assure their initial metallic state. Two other series of $\mathrm{Ru}$ catalyst were prepared, using $\mathrm{RuCl}_{3}$ as precursor (series denoted as "Cl") or using $\mathrm{Ru}(\mathrm{NO})\left(\mathrm{NO}_{3}\right)_{3}$ (series labelled with "NN"). For these two series the graphenic materials were impregnated by incipient wetness method. The metal precursors were dissolved, in both cases, into a water : ethanol (1:1) solution. After evaporation of solvent by keeping the solids overnight in an open recipient at room temperature, the samples were dried at $100{ }^{\circ} \mathrm{C}$ for $24 \mathrm{~h}$. Finally the catalysts were activated by reduction under hydrogen flow as indicated before. Once the reduced samples are at room temperature a helium flow $\left(50 \mathrm{~mL} \mathrm{~min}{ }^{-1}\right)$ is passed for $5 \mathrm{~h}$ in order to passivate the metallic surfaces. The reduced/passivated catalysts were exposed and stored under air up to their evaluation in reaction or the characterization studies.

\section{Material characterizations}

The chemical analysis ( $\mathrm{C}, \mathrm{H}$ and $\mathrm{N}$ ) of the supports was performed using a Perkin-Elmer elemental analyzer. The textural characterization and surface area $\left(S_{\mathrm{BET}}\right)$ determinations were obtained from the nitrogen adsorption $\left(-196{ }^{\circ} \mathrm{C}\right)$ isotherms, which were obtained using a Micromeritics ASAP model 2020 instrument. To obtain the point of zero charge (PZC) of graphenic materials, the electrophoretic mobility $(\mu) v s$. $\mathrm{pH}$ of the samples was measured in a Zeta Meter $3.0+$ at $25{ }^{\circ} \mathrm{C}$. PZC was determined following the experimental procedure described in detail elsewhere. ${ }^{29}$ Transmission Electron Microscopy (TEM) micrographs of the supports and of the catalysts were obtained on a JEOL JEM-2100F microscope at $200 \mathrm{kV}$. The samples were ultrasonically suspended in ethanol before deposition over a carbon-coated copper grid of 200 mesh. The average metal particle sizes in the catalysts were calculated using the following formula: ${ }^{30}$

$$
d=\frac{\sum n_{i} d_{i}^{3}}{\sum n_{i} d_{i}^{2}}
$$

where $n_{i}$ is the number of particles with diameter $d_{i}$.

The samples were also analysed by X-ray diffraction (XRD), using a Polycristal X'Pert Pro PANalytical diffractometer with Nifiltered $\mathrm{Cu} / \mathrm{K}$ radiation $(\lambda=1.54 \AA)$ operating at $45 \mathrm{kV}$ and 40 $\mathrm{mA}$. For each sample, Bragg's angles between $4^{\circ}$ and $90^{\circ}$ were scanned at a rate of $0.04^{\circ} \mathrm{s}^{-1}$. Thermal analysis were also recorded in TG/DTA mode in a TA Instruments SDT Q600 under air. All the catalysts were analysed by X-ray photoelectron spectroscopy (XPS) using an SPECS GmbH with UHV system, energy analyzer PHOIBOS 150 9MCD using a monochromatic Xray source of $\mathrm{Al} \mathrm{K} \alpha(1486.74 \mathrm{eV})$. Each sample was pressed into a small pellet of $10 \mathrm{~mm}$ diameter, placed in the sample holder and degassed in the chamber for $24 \mathrm{~h}$ to achieve a dynamic vacuum below $10^{-10}$ mbar before analysis. The catalysts were first activated by reduction in $\mathrm{H}_{2}$ flow $\left(70 \mathrm{~mL} \mathrm{~min}^{-1}\right)$ at $400{ }^{\circ} \mathrm{C}$ during $1 \mathrm{~h}$ in the sample pretreatment chamber. The spectral data for each sample were analyzed using CASA XPS software. The $\mathrm{C} 1 \mathrm{~s}$ peak at $284.6 \mathrm{eV}$ was used as an internal standard. The equipment error in the energy determinations is less than $0.01 \mathrm{eV}$.

\section{Reactivity measurements}

The oxidation of $5 \mathrm{HMF}$ was carried out using an autoclave (Autoclave Engineers) reactor with $150 \mathrm{~mL}$ capacity, equipped with a mechanical stirrer (500 rpm) and furnace system. By some preliminary studies using the same catalyst it was determined that under this stirring velocity there is not intern mass transfer limitations. After reduction treatment, the catalysts $(50 \mathrm{mg}$ ) were suspended in $100 \mathrm{~mL}$ of water. Then the autoclave was purged three times with synthetic air and the temperature was increased to $100{ }^{\circ} \mathrm{C}$. Once the reaction conditions were reached, $1 \mathrm{~mL}$ of $\mathrm{HMF} 0.2 \mathrm{M}$ in $\mathrm{H}_{2} \mathrm{O}$ was dosed into the autoclave and pressure was raised up to 10 bars with air. Aliquots of the reactor liquids were collected periodically, filtered and the reaction product mixture was analysed by HPLC (Agilent Technologies 1200 series equipped with a refractive index detector, Hi-Plex $\mathrm{H}$ column, flow 0.7 $\mathrm{mL} \min ^{-1}$, mobile phase $5 \mathrm{mM} \mathrm{H}_{2} \mathrm{SO}_{4}$, temperature $65{ }^{\circ} \mathrm{C}$ ). Carbon mass balances in the reaction studies are higher than $94 \%$ in all the catalytic determinations. Some of these experiments were repeated twice in order to check reproducibility of these measurements.

The conversion of HMF was calculated as:

$$
C(\%)=\frac{\mathrm{HMF}_{\mathrm{i}}-\mathrm{HMF}_{\mathrm{f}}}{\mathrm{HMF}_{\mathrm{i}}} \times 100
$$

where $\mathrm{HMF}_{\mathrm{i}}$ represents the initial $\mathrm{HMF}$ concentration and $\mathrm{HMF}_{\mathrm{f}}$ the final HMF concentration.

The selectivity of each product was calculated as:

$$
S_{\mathrm{i}}(\%)=\frac{\mathrm{mol}_{\mathrm{i}}}{\mathrm{mol}_{\mathrm{t}}} \times 100
$$

where $\mathrm{mol}_{\mathrm{i}}$ represents the mole of the product whose selectivity is being calculated and $\mathrm{mol}_{\mathrm{t}}$ represents the total moles of all the products.

Site time yields (STY), moles of FDCA produced per mol of catalyst surface per second, were also calculated. For determine the active surface area exposed by the $\mathrm{Ru}$ nanocrystallites we have assumed perfectly spherical metallic shapes. Thus with the direct measured of the diameters of such as metallic particles by TEM it is possible to determine the numbers of exposed active sites. 


\section{Results and discussion}

\section{Characteristics of the supports}

Fig. 1 shows the XRD patterns of GO, rGO and NrGO. After thermal treatment, the characteristic diffraction peak at $16^{\circ}$ of GO disappeared and a new main peak appeared at $26^{\circ}$ corresponding to the graphite (002) reflection. ${ }^{25}$ The GO was successfully exfoliated to form NrGO and rGO materials. However, the more intense peak at $26^{\circ}$ for NrGO may indicate some restacking of graphene layers. The comparison of this peak for NrGO and rGO samples evidenced that restacking is favored under ammonia reactive conditions. It was also confirmed by $S_{\mathrm{BET}}$ values, so $S_{\mathrm{BET}}$ of rGO $\left(904 \mathrm{~m}^{2} \mathrm{~g}^{-1}\right)$ is higher than that measured for NrGO $\left(483 \mathrm{~m}^{2} \mathrm{~g}^{-1}\right)$ and the degree of restacking should decrease the surface area of graphenic material. ${ }^{25}$ This is consistent with TEM images (Fig. S1 in ESI $\dagger$ ) where it can be observed that graphitic structures appear in the case of NrGO, while rGO solid consists of few layer graphene.

The concentration of nitrogen adatoms in the graphenic supports was determined by elemental analysis. As expected, no nitrogen is detected in rGO. The $\mathrm{N}$ content for NrGO was 4.6 wt $\%$. Furthermore, XPS analyses for the NrGO sample showed nitrogen content of $3.8 \mathrm{wt} \%$ suggesting a somewhat lower concentration of nitrogen species at surface. Deconvolution of the $\mathrm{N} 1 \mathrm{~s}$ region (Fig. S2 in ESI $\dagger$ ) indicated the presence of four nitrogen species: $41 \%$ pyridinic nitrogen (398.5 eV), 27\% pyrrolic nitrogen (400.5 eV), 18\% quaternary nitrogen (401.8 $\mathrm{eV}$ ), and $14 \% \mathrm{NO}_{x}$ groups $(405.0 \mathrm{eV}) .{ }^{31}$ Among all these species, the pyridinic nitrogen is believed to have the stronger basic character. Accordingly, it is generally assumed that the basicity of carbon catalysts is linked to the amount of pyridinic groups. ${ }^{32,33}$ No nitrogen peak was detected for rGO.

As the surface charge of carbonaceous materials is governed by the nature of the surface groups and the $\mathrm{pH}, \mathrm{PZC}$ can be used to estimate the surface chemistry of the graphenic materials. ${ }^{34}$

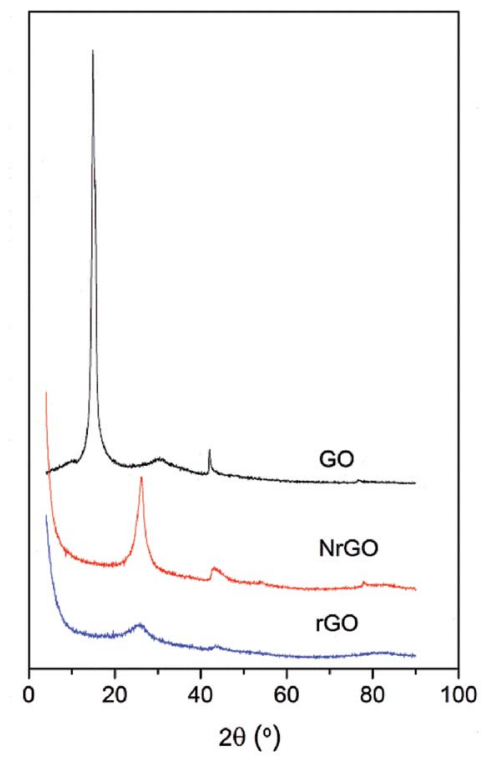

Fig. 1 XRD patterns of GO, NrGO and rGO.
An increase in the PZC value was found for NrGO sample (Fig. 2) showing a PZC of 8.5 against 7.2 of rGO sample. These results indicate that NrGO surfaces present a greater basic character than rGO. Since nitrogen atoms have an additional electron in comparison with carbon atoms, $\mathrm{p}$ electron delocalization will occur easily in NrGO. This excess of electrons produces an increase in the electronic density, which could explain the higher PZC values obtained for NrGO. As electron-donor properties are related to basicity, the stronger the electron donating is, the greater the basicity. ${ }^{31}$ Finally PZC measurements carried out on the reduced catalysts (i.e. samples $\mathrm{Ru}(\mathrm{CO}) / \mathrm{rGO}$ and $\mathrm{Ru}(\mathrm{CO}) / \mathrm{NrGO})$ do not provide significant differences in comparison with the bare supports. This mean that the possible $\mathrm{Ru}$ anchoring over the surface nitrogen groups cannot be detected from the PZC determinations.

The thermal reactivity under air of the prepared supports was investigated by TGA (Fig. S3 $\mathrm{ESI} \dagger$ ). TG analysis for rGO showed a sharp weight loss near $628^{\circ} \mathrm{C}$ that could be attributed to the oxidation of a well-organized carbon structure. In comparison, sample NrGO showed an oxidation temperature of $671{ }^{\circ} \mathrm{C}$, which is in concordance with previously reported trends ${ }^{35}$ showing that the thermal stability in air increases for nitrogen doped graphenic materials.

\section{Characteristics of the $\mathrm{Ru}$ nanoparticles}

The loading of $\mathrm{Ru}$ in carbon supported metal catalyst was estimated by TGA. Thermogravimetric method consisted in weighing the residues of $\mathrm{RuO}_{2}$ generated after burning away the graphenic support at $850{ }^{\circ} \mathrm{C}$ in air. ${ }^{36}$ Also it was checked that bare supports (rGO and NrGO) produce null amount of residue. The same can be said for the HSAG support material. Contrarily in the case of the commercial CA, in spite of its purification treatment, a small residue weight was determined $(0.24 \mathrm{wt} \%)$, this amount being subtracted for determining the quantitative loading of incorporated Ru. Considering the similarities of the nominal amount of $\mathrm{Ru}$ incorporated and the experimental determinations, we can point out that $\mathrm{Ru}$ is not volatilized under these carbon support burning conditions. The chemical compositions of the catalyst are presented in Table 1.

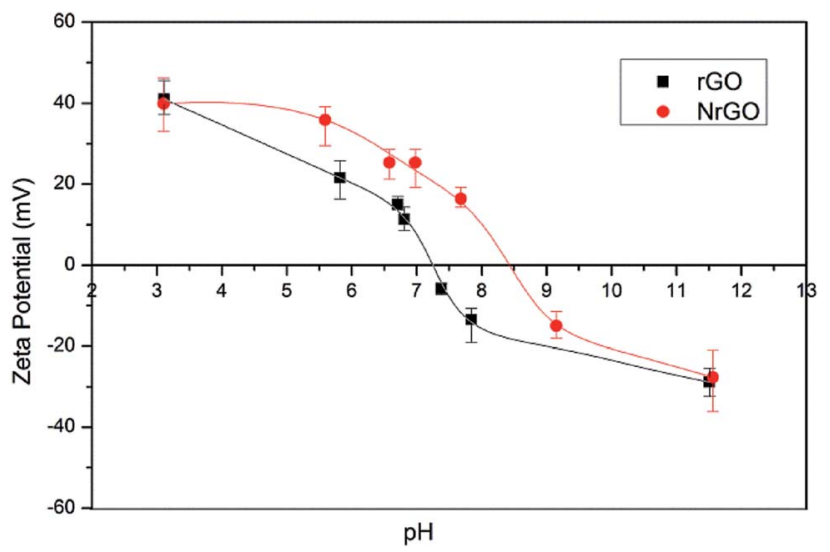

Fig. 2 PZC for rGO and NrGO samples. 
Table 1 Characteristics of the ruthenium particles after reducing samples under hydrogen flow at $350^{\circ} \mathrm{C}$ for $2 \mathrm{~h}$

\begin{tabular}{lll}
\hline Catalyst & $\mathrm{Ru}(\mathrm{wt} \%)$ & $d_{\text {TEM }}(\mathrm{nm})$ \\
\hline $\mathrm{Ru}(\mathrm{Cl}) / \mathrm{rGO}$ & 3.9 & 1.5 \\
$\mathrm{Ru}(\mathrm{Cl}) / \mathrm{NrGO}$ & 4.4 & 2.1 \\
$\mathrm{Ru}(\mathrm{NN}) / \mathrm{rGO}$ & 4.0 & 1.4 \\
$\mathrm{Ru}(\mathrm{NN}) / \mathrm{NrGO}$ & 4.0 & 1.7 \\
$\mathrm{Ru}(\mathrm{CO}) / \mathrm{rGO}$ & 4.3 & 1.4 \\
$\mathrm{Ru}(\mathrm{CO}) / \mathrm{NrGO}$ & 3.9 & 1.8 \\
$\mathrm{Ru}(\mathrm{CO}) / \mathrm{AC}$ & 4.1 & 2.4 \\
$\mathrm{Ru}(\mathrm{CO}) / \mathrm{HSAG}$ & 4.1 & 2.3
\end{tabular}

The XRD patterns of the catalysts prepared from different ruthenium precursors are shown in Fig. 3. All the samples showed the characteristic (002) reflection of graphitic carbon at $26^{\circ}$ (discussed above). However, no peaks related with the formation of crystalline Ru were observed on the samples. This is surely due to the particle size of Ru-NPs in these samples was below the XRD detection threshold as was evidenced in the $d_{\mathrm{TEM}}$ values reported in Table 1 . Finally the addition of metal seems not to change significantly the peak intensity at 26 degree, so the initial structures graphenic or graphitic seems not to be modified by the incorporation of the Ru nanoparticles.

The particle sizes of the ruthenium crystallites exposed in the reduced catalysts were determined by TEM. Representative TEM images of the catalysts and their histograms with particle size distribution are shown in Fig. 4.

The $\mathrm{Ru}$ nanoparticles average size obtained for the carbon supported catalysts are summarized in Table 1 . The average

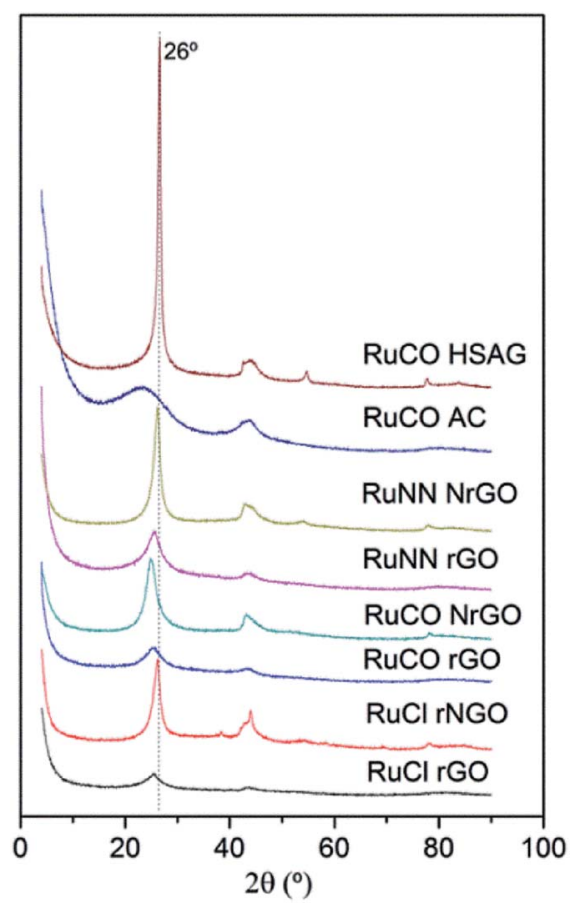

Fig. 3 XRD patterns of $\mathrm{Ru}(\mathrm{Cl}) / \mathrm{rGO}, \mathrm{Ru}(\mathrm{Cl}) / \mathrm{NrGO}, \mathrm{Ru}(\mathrm{NN}) / \mathrm{rGO}$, $\mathrm{Ru}(\mathrm{NN}) / \mathrm{NrGO}, \mathrm{Ru}(\mathrm{CO}) / \mathrm{rGO}, \mathrm{Ru}(\mathrm{CO}) / \mathrm{NrGO}, \mathrm{Ru}(\mathrm{CO}) / \mathrm{AC}$ and $\mathrm{Ru}(\mathrm{CO}) /$ $\mathrm{HSAG}$ after $\mathrm{H}_{2}$ reduction at $350^{\circ} \mathrm{C}$.

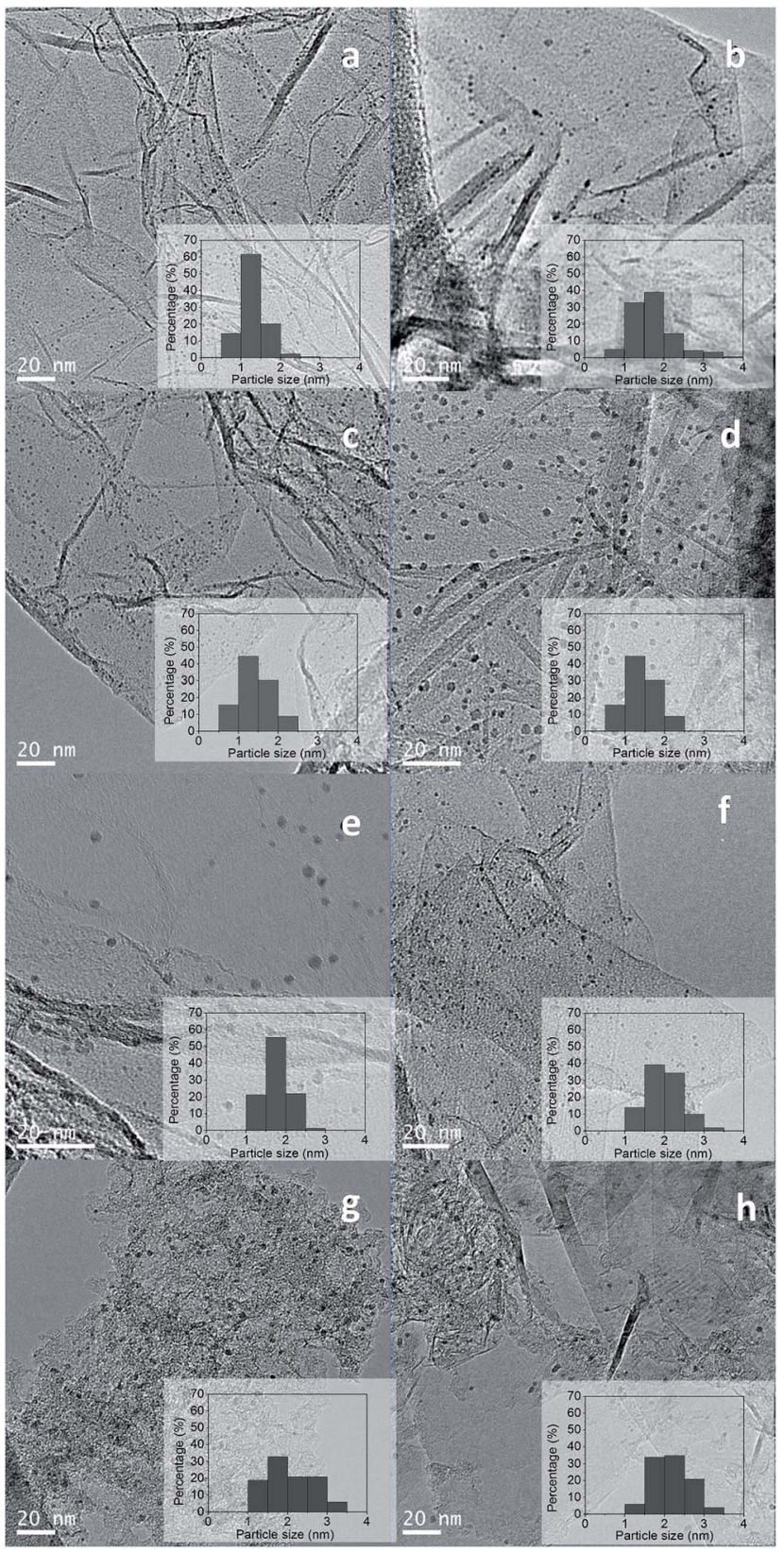

Fig. 4 TEM images and particle size distribution of the catalysts: (a) $\mathrm{Ru}(\mathrm{Cl}) / \mathrm{rGO}$, (b) $\mathrm{Ru}(\mathrm{Cl}) / \mathrm{NrGO}$, (c) $\mathrm{Ru}(\mathrm{NN}) / \mathrm{rGO}$, (d) $\mathrm{Ru}(\mathrm{NN}) / \mathrm{NrGO}$, (e) $\mathrm{Ru}(\mathrm{CO}) / \mathrm{rGO}$, (f) $\mathrm{Ru}(\mathrm{CO}) / \mathrm{NrGO}$, (g) $\mathrm{Ru}(\mathrm{CO}) / \mathrm{AC}$ and (h) $\mathrm{Ru}(\mathrm{CO}) / \mathrm{HSAG}$ after $\mathrm{H}_{2}$ reduction at $350{ }^{\circ} \mathrm{C}$.

ruthenium particle sizes for these catalysts are in the range from 1.4 to $2.4 \mathrm{~nm}$. From Table 1 it should be noted that the particle size of Ru nanoparticles strongly depends from the Ru precursor and the used support. Comparison of catalysts supported on the two graphene supports (rGO and $\mathrm{NrGO}$ ) for a given $\mathrm{Ru}$ precursor reveals that larger particle sizes were systematically obtained on NrGO support, which can be rationalized in terms of the lower specific surface area of the NrGO support regarding rGO support. When a graphene support (rGO or $\mathrm{NrGO}$ ) is considered the ruthenium particle size change with the metal precursor in the order: $\mathrm{Ru}(\mathrm{Cl})>\mathrm{Ru}(\mathrm{NN}) \approx \mathrm{Ru}(\mathrm{CO})$. As 
shown in Fig. $4 \mathrm{Ru}$ nanoparticles were dispersed uniformly on graphene surfaces without detecting aggregation formation, so the interactions of graphene surfaces with the Ru crystallites seem to be maximized. On the other hand, there are not evidences of any association of the Ru nanoparticles with the $\mathrm{N}$ atoms of the NrGO support, and these nitrogen functionalities seem not to play any role in determining the Ru crystallite sizes. In general mean $\mathrm{Ru}$ particle sizes (Table 1) are higher in the catalysts supported over $\mathrm{NrGO}$ than in those supported over rGO. The diameter size distribution histograms of these catalysts are displayed in Fig. 4 . It is observed that the particle size distribution is broadened when the NrGO is used as support with respect to $\mathrm{rGO}$. Moreover, $\mathrm{Ru}(\mathrm{CO}) / \mathrm{HSAG}$ and $\mathrm{Ru}(\mathrm{CO}) / \mathrm{AC}$ catalysts present the highest average particle size and also the broadest particle size distribution. This is likely consequence of the lower surface area of HSAG $\left(396 \mathrm{~m}^{2} \mathrm{~g}^{-1}\right)$ and AC $\left(313 \mathrm{~m}^{2} \mathrm{~g}^{-1}\right.$ external surface area) compared to rGO $\left(904 \mathrm{~m}^{2} \mathrm{~g}^{-1}\right)$ and $\mathrm{NrGO}$ $\left(483 \mathrm{~m}^{2} \mathrm{~g}^{-1}\right)$.

XPS analysis was done in order to study the electronic states of Ru nanoparticle on the different catalysts. Due to the binding energy of $\mathrm{Ru} 3 \mathrm{~d}_{3 / 2}$ overlapped partially with that of $\mathrm{C} 1 \mathrm{~s}, \mathrm{Ru} 3 \mathrm{p}$ signal was used to distinguish the chemical states of $\mathrm{Ru}$ samples. The $\mathrm{Ru} 3 \mathrm{p}$ XPS spectrum for $\mathrm{Ru}(\mathrm{Cl}) / \mathrm{NrGO}$ catalyst showed two peaks centered at $462.7 \mathrm{eV}$ and $484.7 \mathrm{eV}$ corresponding to $\mathrm{Ru} 3 \mathrm{p}_{3 / 2}$ and $\mathrm{Ru} 3 \mathrm{p}_{1 / 2}$ respectively (Table 2 and Fig. 5a). Notably, the peaks for $\mathrm{Ru}(\mathrm{Cl}) / \mathrm{NrGO}$ appeared at lower binding energy compared to $\mathrm{Ru}(\mathrm{Cl}) / \mathrm{rGO}$ peaks $(463.0 \mathrm{eV}$ and $485.0 \mathrm{eV}$ in Table 2). The latter fact suggests that the incorporation of nitrogen atoms in the graphitic structure of graphene could favor donation of electron density towards Ru active sites. More significantly some residual chlorine impurities on the surface were observed in the survey scan XPS spectrum of $\mathrm{Ru}(\mathrm{Cl}) / \mathrm{NrGO}$ and $\mathrm{Ru}(\mathrm{Cl}) / \mathrm{rGO}$ catalysts. However some similar shifts in the Ru binding energies towards lower energy values were observed when compared with $\mathrm{Ru}(\mathrm{CO}) / \mathrm{NrGO}$ and $\mathrm{Ru}(\mathrm{NN}) /$ NrGO catalysts (Table 2 and Fig. S4 $\uparrow$ ). That we can conclude that when the $\mathrm{Ru}$ nanoparticles are supported over the nitrogendoped graphenic material there is a systematic electronic interaction between this support and the Ru nanoparticles with electron transfer from the support to the Ru. In agreement with our results an earlier work of X. Chen et $\mathrm{al}^{37}$ reported that the Ndoped graphene is an effective electron donor for iron nanoparticles as revealed by Fe K-edge XANES study.

\section{Catalytic results}

Previous research with different metals (Ru, Pt) and supports (oxidic and carbonaceous materials), ${ }^{19,21}$ demonstrates both the

Table 2 XPS data of Ru catalysts

\begin{tabular}{llll}
\hline Catalyst & BE Ru 3p $\mathrm{p}_{3 / 2}(\mathrm{eV})$ & FWHM & $\mathrm{Ru} / \mathrm{C}$ \\
\hline $\mathrm{Ru}(\mathrm{Cl}) / \mathrm{rGO}$ & 463.0 & 2.4 & 0.001 \\
$\mathrm{Ru}(\mathrm{Cl}) / \mathrm{NrGO}$ & 462.7 & 3.1 & 0.002 \\
$\mathrm{Ru}(\mathrm{NN}) / \mathrm{NrGO}$ & 462.5 & 3.6 & 0.004 \\
$\mathrm{Ru}(\mathrm{CO}) / \mathrm{NrGO}$ & 462.4 & 3.5 & 0.005
\end{tabular}

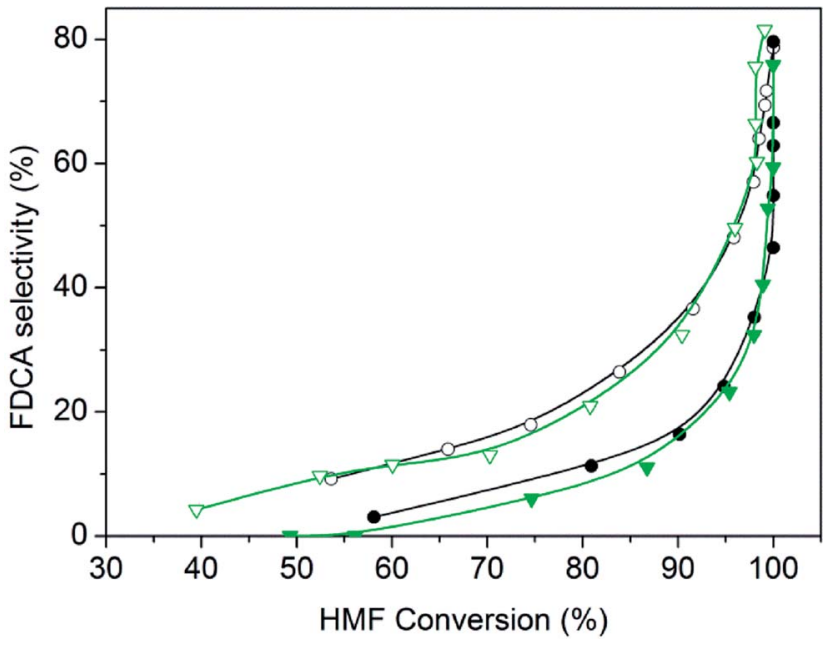

Fig. 5 Evolution of selectivity towards FDCA during HMF oxidation reaction over Ru nanoparticles supported on nitrogen functionalized graphenic material: (o) $\mathrm{Ru}(\mathrm{CO}) / \mathrm{NrGO},(\nabla) \mathrm{Ru}(\mathrm{NN}) / \mathrm{NrGO}$ or on undoped graphene: $(\bullet) \mathrm{Ru}(\mathrm{CO}) / \mathrm{rGO},(\nabla) \mathrm{Ru}(\mathrm{NN}) / \mathrm{rGO}$. Reaction conditions: $\mathrm{HMF}, 2 \mathrm{mmol}, 50 \mathrm{mg}$ of catalyst, molar ratio $\mathrm{HMF} / \mathrm{M}=10, \mathrm{H}_{2} \mathrm{O} 100 \mathrm{~mL}$, air 10 bars, $100{ }^{\circ} \mathrm{C}, 8$ hours.

relevance of the support in the achieved final FDCA yield (Scheme 1), and that in general carbon supports are superior.

Table 3 lists the results of the base-free aqueous-phase oxidation of HMF to FDCA over the supported Ru catalysts prepared from different precursors. First, blank tests were carried out, without catalyst addition in the reactor, and barely formation of products was observed (entry 1 Table 3). Also the bare supports give very low HMF conversions (entries 2 and 3) under our reaction conditions. From Table 3 it can be observed that the achieved HMF conversions with nearly all the $\mathrm{Ru}$ studied catalysts were higher than $97.5 \%$ after $8 \mathrm{~h}$ in reaction. Concerning the selectivity values significant differences can be observed depending on precursor and support. For instance when Ru nanoparticles are supported on commercial carbons $(\mathrm{Ru}(\mathrm{CO}) / \mathrm{HSAG}$ and $\mathrm{Ru}(\mathrm{CO}) / \mathrm{AC})$ FDCA selectivity values are significantly lower than when supported on graphenic materials. For $\mathrm{Ru}(\mathrm{CO}) / \mathrm{AC}$ and $\mathrm{Ru}(\mathrm{CO}) / \mathrm{HSAG}$ catalysts the poor

Table 3 Catalytic performance of ruthenium catalysts in the oxidation of HMF

\begin{tabular}{|c|c|c|c|c|c|}
\hline Entry & Catalyst & $\begin{array}{l}\text { Conversion } \\
(\%)\end{array}$ & $\begin{array}{l}\text { Sel FDCA } \\
(\%)\end{array}$ & $\begin{array}{l}\text { Sel FFCA } \\
(\%)\end{array}$ & $\begin{array}{l}\text { Sel DFF } \\
(\%)\end{array}$ \\
\hline 1 & Blank & 1.6 & 0.0 & 0.0 & 100.0 \\
\hline 2 & NrGO & 4.2 & 0.0 & 16.5 & 83.5 \\
\hline 3 & $\mathrm{rGO}$ & 2.7 & 0.0 & 0.0 & 100 \\
\hline 4 & $\mathrm{Ru}(\mathrm{Cl}) / \mathrm{rGO}$ & 99.1 & 49.4 & 47.5 & 3.1 \\
\hline 5 & $\mathrm{Ru}(\mathrm{Cl}) / \mathrm{NrGO}$ & 97.5 & 43.1 & 48.0 & 8.8 \\
\hline 6 & $\mathrm{Ru}(\mathrm{NN}) / \mathrm{rGO}$ & 100.0 & 75.8 & 24.2 & 0.0 \\
\hline 7 & $\mathrm{Ru}(\mathrm{NN}) / \mathrm{NrGO}$ & 99.1 & 81.5 & 18.0 & 0.5 \\
\hline 8 & $\mathrm{Ru}(\mathrm{CO}) / \mathrm{rGO}$ & 100.0 & 79.6 & 20.4 & 0.0 \\
\hline 9 & $\mathrm{Ru}(\mathrm{CO}) / \mathrm{NrGO}$ & 100.0 & 79.1 & 20.9 & 0.0 \\
\hline 10 & $\mathrm{Ru}(\mathrm{CO}) / \mathrm{HSAG}$ & 99.3 & 40.5 & 53.6 & 5.9 \\
\hline 11 & $\mathrm{Ru}(\mathrm{CO}) / \mathrm{AC}$ & 99.2 & 39.0 & 59.0 & 2.1 \\
\hline
\end{tabular}


catalytic selectivities towards FDCA can be attributed to the Ru average particle sizes, $2.3 \mathrm{~nm}$ and $2.4 \mathrm{~nm}$ respectively (Table 1), which are significantly higher than the sizes in Ru catalysts supported on graphene supports (rGO and N-rGO), in particular than those prepared with $\mathrm{Ru}$ carbonyl as metal precursor. Apparently an increased average metal particle size implies a reduction in the reaction rate since the progress of the consecutive oxidation reactions to reach the desired FDCA product is slower.

The selectivities toward FDCA obtained with catalysts prepared from $\mathrm{RuCl}_{3}$ precursor over graphenic materials were also lower than selectivity values obtained with $\mathrm{Ru}_{3}(\mathrm{CO})_{12}$ and $\mathrm{Ru}(\mathrm{NO})\left(\mathrm{NO}_{3}\right)_{3}$ derived catalysts. The presence of chlorine impurities, that was confirmed by XPS as it was discussed above, can be the reason of this different behavior. This poisoning of the Ru nanoparticles ${ }^{38}$ and a possible increase of acidity due to the presence of chlorine ions, seems to be responsible for the low selectivity towards FDCA of $\mathrm{Ru}(\mathrm{Cl}) / \mathrm{rGO}$ and $\mathrm{Ru}(\mathrm{Cl}) / \mathrm{NrGO}$ catalysts.

For catalysts prepared with both $\mathrm{Ru}_{3}(\mathrm{CO})_{12}$ and $\mathrm{Ru}(\mathrm{NO})\left(\mathrm{NO}_{3}\right)_{3}$ precursors, higher selectivity towards FDCA was found when supported on NrGO in comparison with those supported on rGO. In Fig. 5 the evolution of selectivity towards FDCA as the reaction is progressing is represented. Clearly a systematic support effect is evidenced. For these four catalysts, with $\mathrm{Ru}$ average particle sizes among $1.4 \mathrm{~nm}$ and $1.8 \mathrm{~nm}$, as reported in Table 1, the possible effect of the nitrogen surface groups can be neatly evidenced. The single most noteworthy observation from these comparative data was that HMF could be completely converted, and with $79.1 \%$ selectivity towards FDCA, over $\mathrm{Ru}(\mathrm{CO}) / \mathrm{NrGO}$ after $8 \mathrm{~h}$ at $100{ }^{\circ} \mathrm{C}$ (entry 9).

In order to quantitatively compare the effects induced by the presence of nitrogen surface groups in the graphenic materials when used as supports, in Fig. 6 catalyst activity is reported for

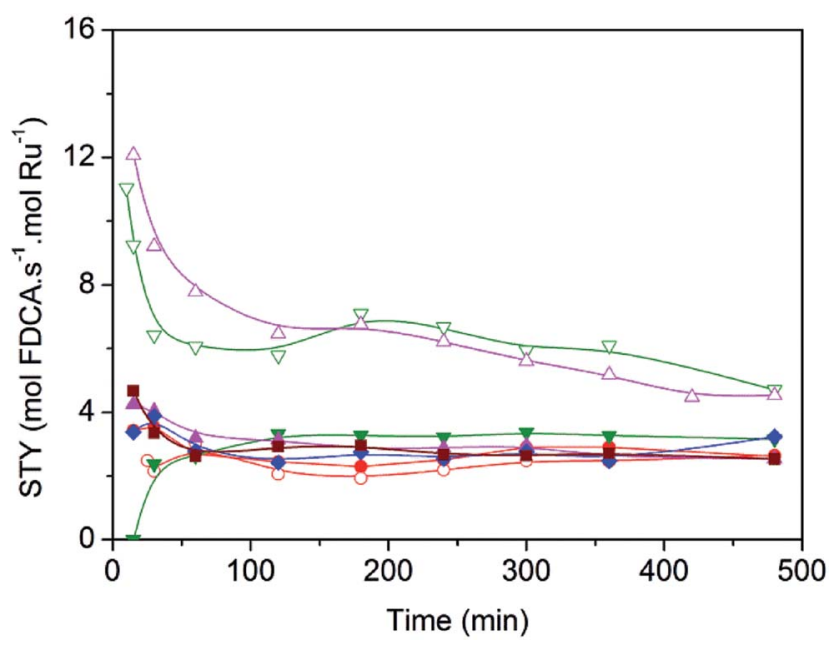

Fig. 6 Site time yield for FDCA (STY, mol per s per mol $r$ Ru) with time of reaction (min) over (๑) Ru(Cl)/rGO, (O) Ru(Cl)/NrGO, ( $) \mathrm{Ru}(\mathrm{NN}) / \mathrm{rGO}$, $(\nabla) \mathrm{Ru}(\mathrm{NN}) / \mathrm{NrGO},(\Delta) \mathrm{Ru}(\mathrm{CO}) / \mathrm{rGO},(\triangle) \mathrm{Ru}(\mathrm{CO}) / \mathrm{NrGO},(\diamond) \mathrm{Ru}(\mathrm{CO}) / \mathrm{AC}$ and (口) Ru(CO)/HSAG. Reaction conditions: HMF, 2 mmol, $50 \mathrm{mg}$ of catalyst, molar ratio $\mathrm{HMF} / \mathrm{M}=10, \mathrm{H}_{2} \mathrm{O} 100 \mathrm{~mL}$, air 10 bars, $100^{\circ} \mathrm{C}, 8$ hours. each catalyst as site time yield (STY), moles of FDCA produced per mol of surface Ru per second. Clearly the two Ru catalysts supported on Nr-GO produce, in all the range of reaction time, higher selectivity values towards FDCA formation than those supported on rGO. This effect is observable for the two series of catalysts: ex-nitrosyl nitrate and ex-carbonyl. As reported in Table 3, entries 2 and 3, the bare support NrGO produce significant higher amount of FFCA in comparison with rGO, in spite of the low HMF conversion (Table 3). This FFCA is an intermediated in the formation of FDCA (Scheme 1).

Therefore, all these results would seem to indicate the nitrogen functional groups significantly affect in the NrGO supported $\mathrm{Ru}$ catalysts, improving the selectivity to FDCA. The nature of the nitrogen effect could be thought to be twofold. On the one hand, the basic sites, as evidenced from PZC (Fig. 2), in the case of $\mathrm{Ru}$ catalysts supported on $\mathrm{NrGO}$, surely may contribute to the reaction in synergy with the Ru nanoparticles. However, the electron enrichment of the $\mathrm{Ru}$ nanoparticles, observed by XPS measurements, which could affect their activity for the HMF oxidation, can not improve the activity for the oxidation reaction, because a higher electron density of the surface $\mathrm{Ru}$ would disfavor both the dioxygen dissociation and the HMF chemisorption. Thus our main hypothesis is that basic sites, in the case of Ru nanoparticles supported on NrGO, surely may contribute to the reaction in synergy with the $\mathrm{Ru}$ nanoparticles. In short the combination of very small Ru crystallites
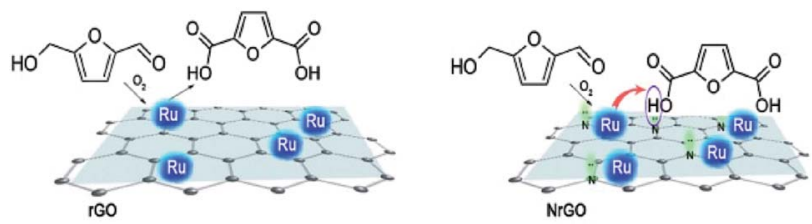

Scheme 2 Proposal for the cooperative action of the nitrogen surface groups exposed on the doped graphenic materials and the $\mathrm{Ru}$ nanoparticles.

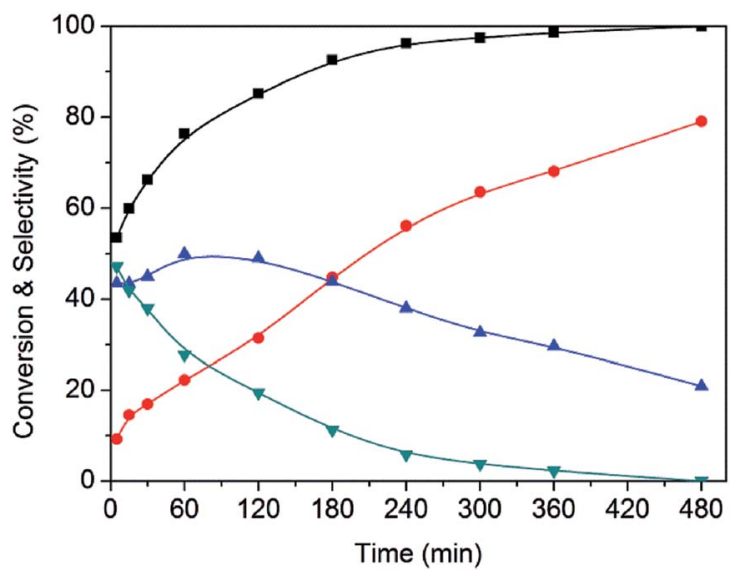

Fig. 7 Time course of product formation for HMF oxidation over

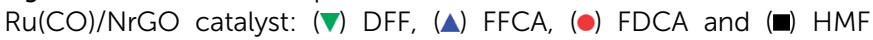
conversion. Reaction conditions: HMF, $2 \mathrm{mmol}, 50 \mathrm{mg}$ of catalyst, molar ratio $\mathrm{HMF} / \mathrm{M}=10, \mathrm{H}_{2} \mathrm{O} 100 \mathrm{~mL}$, air 10 bars, $100^{\circ} \mathrm{C}, 8$ hours. 


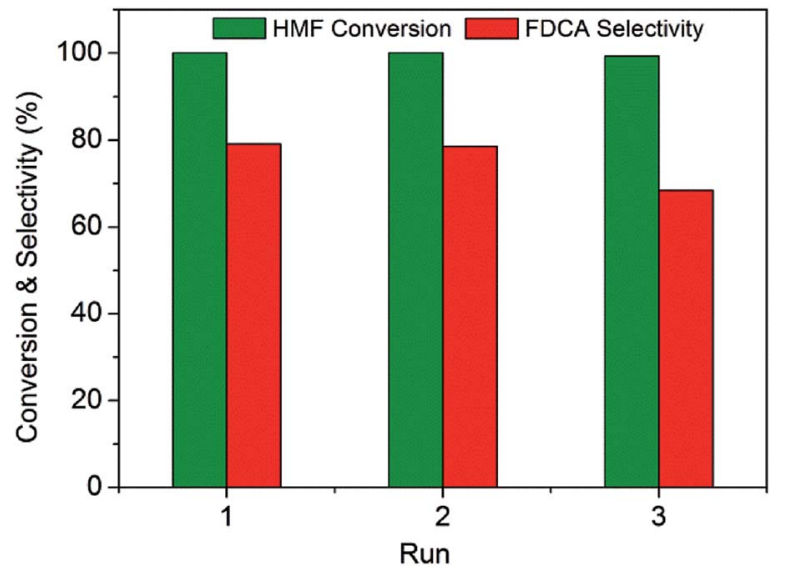

Fig. 8 Stability of the $\mathrm{Ru}(\mathrm{CO}) / \mathrm{NrGO}$ catalyst during the recycling uses for the oxidation of HMF: (green bar) HMF conversion, (red bar) FDCA selectivity. Reaction conditions: HMF, $2 \mathrm{mmol}, 50 \mathrm{mg}$ of catalyst, molar ratio $\mathrm{HMF} / \mathrm{M}=10, \mathrm{H}_{2} \mathrm{O} 100 \mathrm{~mL}$, air 10 bars, $100^{\circ} \mathrm{C}, 8$ hours.

with some basic surface functions can act cooperatively. In a first stage oxygen and reactant are chemisorbed on the $\mathrm{Ru}$ surface and the production of acidic compounds (HFCA, FFCA and FDCA) starts. When FDCA is formed this is more efficiently removed from the metallic surface in the presence of basic sites exposed on the graphenic materials. These mechanistic aspects have been outlined in the Scheme 2, and should be considered as an explanation of the results presented in Fig. 5 and 6. So, this mechanism is close to that taking place when a basic compound is dissolved in the reaction media: reaction equilibrium displacement by elimination of the products from the proximity of the active surface sites. We can define this mechanism action as a cooperative effect between the surface sites exposed on the Ru nanoparticles and those basic functionalities exhibited on the nitrogen doped graphenic material (NrGO).

A representative example of the time course of the conversion (\%) of HMF to the reaction products (\%), determined using the $\mathrm{Ru}(\mathrm{CO}) / \mathrm{NrGO}$ catalyst, are presented in Fig. 7. During the first 15 minutes, the main product was DFF. As HMF was consumed, the yield of DFF decreased and the yield of FFCA increased. The yield of the intermediates decreased and the yield of FDCA increased up to near $79 \%$ at 8 hours. Furthermore no HMFCA formation was detected. On the basis of these results we proposed that under our experimental conditions FDCA is produced by a stepwise reaction via DFF and FFCA as shown Scheme 1. This tendency is in good agreement with previous studies ${ }^{21}$ in the base-free oxidation of HMF. It is also worth noting that $85 \%$ of HMF is converted in the first two hours showing that the steps HMF to DFF and FFCA are faster than the reaction of transformation of FFCA into FDCA. Therefore, $\mathrm{Ru}$ catalyzed the oxidation of $-\mathrm{OH}$ to $-\mathrm{CHO}$, and further oxidized to $-\mathrm{COOH}$, this latter being the rate-limiting step.

In order to evaluate the stability of this $\mathrm{Ru}(\mathrm{CO}) / \mathrm{NrGO}$ catalytic material, three successive rounds were carried out with the solid recovered by filtration and washed with water. As shown in Fig. 8, the initial conversion of HMF was maintained for at least three runs. However, the selectivity of FDCA slightly decreased

Table 4 Comparative data of HMF oxidation over different metal supported catalysts

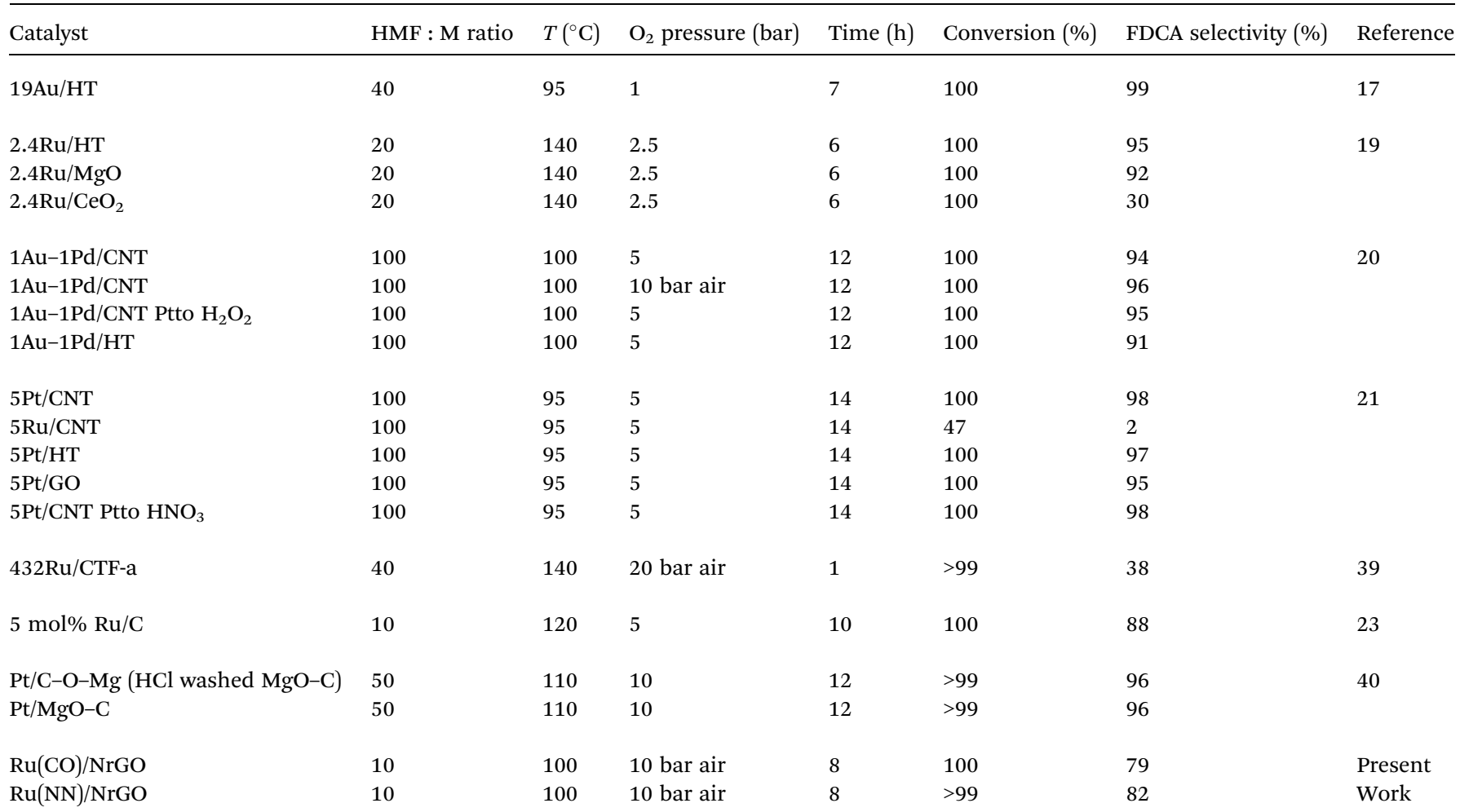


during the recycle process. It could be related to a small loss of mass during recovery of the catalyst between cycles and/or with a blocking of the support basic surface sites by some adsorbed reaction products. Finally the concentration of potentially leached ruthenium in the aqueous solution was analysed by inductively coupled plasma mass spectrometer (ICP-MS). For post-reaction solutions obtained from the $\mathrm{Ru}(\mathrm{CO}) / \mathrm{NrGO}$ catalyst after the three stability runs, no ruthenium was detected. This absence of Ru leaching can be rationalized considering that before reaction tests catalysts were subjected to a reduction pretreatment at $350{ }^{\circ} \mathrm{C}$, and the resulting metallic $\mathrm{Ru}$ particles are no soluble in water media. Thus all these results demonstrated the outstanding stability and reusability of our improved $\mathrm{Ru}(\mathrm{CO}) / \mathrm{NrGO}$ catalyst.

In order to compare our best performance catalysts with those previously published, in Table 4 are reported data obtained using different noble metal catalysts supported over diverse supports. It should be notice that in any case presented data are obtained using added base as co-catalyst and in all the reactions presented in Table 4 water is the solvent media. So these reported data are obtained under the greenest conditions. The comparison among catalysts in Table 4 is not easy since the reaction variables, such as temperature, pressure, reaction time or molar reactant-metal ratio, are not identical. However, our $\mathrm{Ru}$ catalysts are similar in terms of activity and selectivity to those reported in the literature containing noble metals (Au, Pt) which are more expensive than $\mathrm{Ru}$. When comparing with $\mathrm{Ru}$ catalysts supported on different materials, our samples result slightly superior, just considering that we work at lower reaction temperature and pressure.

\section{Conclusions}

Ru catalysts prepared using different metal precursors (nitrosyl nitrate, carbonyl and chloride) and supported over various carbonaceous materials (high surface area graphite, activated carbon, graphene and $\mathrm{N}$-doped graphene) were studied in the aqueous-phase aerobic oxidation of HMF. The reported data of selectivity cannot be only explained as consequence of $\mathrm{Ru}$ particle sizes, as evidenced by the similar average particle sizes determined by TEM (in the range between 1.4 and $2.4 \mathrm{~nm}$ ). The obtained results suggest that the support along with the $\mathrm{Ru}$ precursor remarkably affect the product distributions and surface properties of the ruthenium nanoparticles over the supports. In particular graphenic materials are superior in comparison with commercial carbonaceous materials. Significantly it was revealed that basic surface nitrogen heteroatoms exposed on the NrGO support can play an important role, particularly in the desired product selectivity. As tentative interpretation of this systematic support effect we have argued that $\mathrm{Ru}$ nanoparticles supported on nitrogen doped graphene can act synergistically with the basic surface nitrogen sites exposed on the doped graphene surface. These latter can remove the acidic products of this reaction cooperating with the maintenance of free surface $\mathrm{Ru}$ sites to perform the oxidation reaction more rapidly.

\section{Conflicts of interest}

There are no conflicts to declare.

\section{Acknowledgements}

CRB gratefully acknowledges financial support from Spanish Ministerio de Educacion, Cultura y Deporte Grant No. FPU15/ 01838. Also the financial support from the Spanish Ministerio de Economía y Competitividad under projects CTQ2014-52956C3-2-R and CTQ2014-52956-C3-3-R is recognized.

\section{Notes and references}

1 M. J. Climent, A. Corma and S. Iborra, Green Chem., 2014, 16(2), 516-547.

2 J. N. Chheda, G. W. Huber and J. A. Dumesic, Angew. Chem. Int. Ed., 2007, 46(38), 7164-7183.

3 T. Werpy and G. Petersen, Top Value Added Chemicals from Biomass, 2004, vol. 1.

4 R. A. Sheldon, Green Chem., 2014, 16(3), 950-963.

5 T. Miura, H. Kakinuma, T. Kawano and H. Matsuhisa. Method for producing furan-2,5-dicarboxylic acid, US pat., 7411078B2, 2008.

6 W. Partenheimer and V. V. Grushin, Adv. Synth. Catal., 2001, 343(1), 102-111.

7 Z. Zhang and K. Deng, ACS Catal., 2015, 5(11), 6529-6544.

8 P. Vinke, H. E. Van Dam and H. Van Bekkum, Stud. Surf. Sci. Catal., 1990, 55(8), 147-158.

9 M. a Lilga, R. T. Hallen and M. Gray, Top. Catal., 2010, 53(1518), 1264-1269.

10 O. Casanova, S. Iborra and A. Corma, ChemSusChem, 2009, 2(12), 1138-1144.

11 B. Donoeva, N. Masoud and P. E. de Jongh, ACS Catal., 2017, 7, 4581-4591.

12 J. Xie, J. Nie and H. Liu, Chin. J. Catal., 2014, 35(6), 937-944. 13 A. a. Rosatella, S. P. Simeonov, R. F. M. Frade and C. A. M. Afonso, Green Chem., 2011, 13(4), 754.

14 S. Albonetti, A. Lolli, V. Morandi, A. Migliori, C. Lucarelli and F. Cavani, Appl. Catal., B, 2015, 163, 520-530.

15 F. Neaţu, R. S. Marin, M. Florea, N. Petrea, O. D. Pavel and V. I. Pârvulescu, Appl. Catal., B, 2016, 180, 751-757.

16 O. Casanova, S. Iborra and A. Corma, J. Catal., 2009, 265(1), 109-116.

17 N. K. Gupta, S. Nishimura, A. Takagaki and K. Ebitani, Green Chem., 2011, 13(4), 824.

18 L. Ardemani, G. Cibin, A. J. Dent, M. A. Isaacs, G. Kyriakou, A. F. Lee, C. M. A. Parlett, S. A. Parry and K. Wilson, Chem. Sci., 2015, 6(8), 4940-4945.

19 Y. Y. Gorbanev, S. Kegnæs and A. Riisager, Top. Catal., 2011, 54(16-18), 1318-1324.

20 X. Wan, C. Zhou, J. Chen, W. Deng, Q. Zhang, Y. Yang and Y. Wang, ACS Catal., 2014, 4(7), 2175-2185.

21 C. Zhou, W. Deng, X. Wan, Q. Zhang, Y. Yang and Y. Wang, ChemCatChem, 2015, 7(18), 2853-2863. 
22 D. Lei, K. Yu, M.-R. Li, Y. Wang, Q. Wang, T. Liu, P. Liu, L.-L. Lou, G. Wang and S. Liu, ACS Catal., 2017, 7(1), 421432.

23 G. Yi, S. P. Teong and Y. Zhang, Green Chem., 2016, 18(4), 979-983.

24 F. F. Tao, Metal Nanoparticles for Catalysis: Advances and Applications, ed. F. Tao, The Royal Society of Chemistry, Cambridge, 2014.

25 E. Asedegbega-Nieto, M. Perez-Cadenas, M. V. Morales, B. Bachiller-Baeza, E. Gallegos-Suarez, I. Rodriguez-Ramos and A. Guerrero-Ruiz, Diamond Relat. Mater., 2014, 44, 2632.

26 E. Castillejos-Lopez, B. Bachiller-Baeza, E. AsedegbegaNieto, A. Guerrero-Ruiz and I. Rodriguez-Ramos, RSC Adv., 2015, 5(99), 81583-81598.

27 G. Bottari, M. Á. Herranz, L. Wibmer, M. Volland, L. Rodríguez-Pérez, D. M. Guldi, A. Hirsch, N. Martín, F. D'Souza and T. Torres, Chem. Soc. Rev., 2017, 46, 4464-4500.

28 B. C. Brodie, Philos. Trans. R. Soc. London, 1859, 149(9), 249259.

29 C. Ramirez-Barria, A. Guerrero-Ruiz, E. Castillejos-Lopez, I. Rodríguez-Ramos, J. Durand, J. Volkman and P. Serp, RSC Adv., 2016, 6(59), 54293-54298.
30 E. Gallegos-Suarez, M. Perez-Cadenas, A. Guerrero-Ruiz, I. Rodriguez-Ramos and A. Arcoya, Appl. Surf. Sci., 2013, 287, 108-116.

31 L. Faba, Y. a. Criado, E. Gallegos-Suarez, M. Pérez-Cadenas, E. Díaz, I. Rodriguez-Ramos, A. Guerrero-Ruiz and S. Ordóñez, Appl. Catal., A, 2013, 458, 155-161.

32 S. Van Dommele, K. P. de Jong and J. H. Bitter, Chem. Commun., 2006, 76(46), 4859-4861.

33 B. Li, X. Sun and D. Su, Phys. Chem. Chem. Phys., 2015, 17, 6691-6694.

34 P. Serp and J. L. Figueiredo, Carbon Materials for Catalysis, ed. P. Serp and J. L. Figueiredo, John Wiley \& Sons, Inc, New Jersey, 2008.

35 R. Canty, E. Gonzalez, C. MacDonald, S. Osswald, H. Zea and C. C. Luhrs, Materials, 2015, 8(10), 7048-7058.

36 A. Guerrero-Ruiz, P. Badenes and I. Rodríguez-Ramos, Appl. Catal., A, 1998, 173(2), 313-321.

37 X. Chen, D. Deng, X. Pan, Y. Hu and X. Bao, Chem. Commun., 2015, 51(1), 217-220.

38 a. Guerrero-Ruiz, E. Gallegos-Suarez, L. Gonzalo-Chacon and I. Rodriguez-Ramos, Thermochim. Acta, 2013, 567, 112-117.

39 J. Artz and R. Palkovits, ChemSusChem, 2015, 8(22), 38323838.

40 X. Han, L. Geng, Y. Guo, R. Jia, X. Liu, Y. Zhang and Y. Wang, Green Chem., 2016, 18, 1597-1604. 